\title{
Parámetros de laboratorio de importancia en el manejo de pacientes con COVID-19
}

\author{
Marion Echenagucía-Echenagucía ${ }^{1,2}$, Rocío Trueba-Gómez ${ }^{2,3}$, Fany Rosenfeld-Mann ${ }^{2,3}$, \\ César Zavala-Hernández ${ }^{2,4}$, Carlos Martínez-Murillo2,5 y Aurora de la Peña-Díaz2,6,7* \\ ${ }^{1}$ Banco Municipal de Sangre, Caracas, Venezuela; ${ }^{2}$ Comité de Trombosis y Hemostasia-AMEH A.C. Ciudad de México, México; ${ }^{3}$ Coordinación de \\ Hematología Perinatal, Instituto Nacional de Perinatología Isidro Espinosa de los Reyes, Ciudad de México, México; ${ }^{4}$ Laboratorio Clínico, Instituto \\ Nacional de Rehabilitación, Ciudad de México, México; ${ }^{5}$ Departamento de Hematología, Hospital General de México Dr. Eduardo Liceaga, Ciudad \\ de México, México; ${ }^{6}$ Departamento de Farmacología, Facultad de Medicina, Universidad Nacional Autónoma de México, Ciudad de México, México; \\ ${ }^{7}$ Departamento de Biología Molecular Instituto Nacional de Cardiología Ignacio Chávez, Ciudad de México, México
}

\section{Resumen}

El coronavirus 2 del síndrome respiratorio agudo grave (SARS-CoV-2) detona el padecimiento la enfermedad por coronavirus 2019 (COVID-19), poniendo en riesgo de muerte a la población vulnerable. El laboratorio clínico enfrenta un reto para el diagnóstico, seguimiento, pronóstico y evaluación de los tratamientos, que se ofrecen a los enfermos de COVID-19. Nuestro objetivo es mostrar al lector un resumen de los principales cambios en los parámetros que se estudian en los laboratorios clínicos. Material y métodos: Se hizo una búsqueda bibliográfica cruzando los términos COVID-19 y laboratorio clínico. Se analizaron las publicaciones relevantes por los miembros del Comité de Trombosis y Hemostasia-AMEH A.C. y se plasmaron las pruebas que a criterio de los participantes destacan por la relación entre la información que proporcionan respecto al seguimiento, pronóstico y evaluación al tratamiento. Resultados: Se recomienda solicitar una citometría hemática (recuento de células blancas, relación neutrófilo:linfocito), química sanguínea (transaminasas, bilirrubinas, albúmina, urea, creatinina, glucosa, lactato deshidrogenasa), pruebas de coagulación (tiempo de protrombina, tiempo de tromboplastina parcial activado, fibrinógeno y dímeros D) y pruebas especiales (proteína C reactiva, ferritina, procalcitonina, troponina).

PALABRAS CLAVE: COVID-19. Laboratorio Clínico. Disfunción endotelial.

\section{Laboratory parameters of importance in the management of COVID-19 patients}

\begin{abstract}
The severe acute respiratory syndrome coronavirus 2 (SARS-CoV-2) triggers the coronavirus disease 2019 (COVID-19), putting the vulnerable population at risk of death. The clinical laboratory faces a challenge for the diagnosis, monitoring, prognosis and evaluation of therapy with low molecular weight heparin. Our objective in this article is to offer a brief discuss of the main changes in the clinical parameters, studied on behalf of COVID-19 patients by a clinical laboratory. Material and methods: $A$ bibliographic search was made crossing the terms COVID-19 and clinical laboratory. Relevant publications were analyzed by the members of the Committee for Thrombosis and Hemostasis-AMEH A.C. The relevant articles were discussed, and the clinical tests discussed in the article are those, that meet the criteria of providing information referring to the follow-up, prognosis and evaluation of treatment against the lower cost. Results: It is recommended to request a blood count (white cell count, neutrophyl/ lymphocytes ratio), clinical chemistry (transaminases, bilirubin, albumin, urea, creatinine, glucose, lactate dehydrogenase), coagulation tests (prothrombin time, activated partial thromboplastin time, fibrinogen, $D D$ dimers) and special tests (C-reactive protein, ferritin, procalcitonin, troponins).
\end{abstract}

KEY WORDS: COVID-19. Clinical Laboratory. Endothelial dysfunction.

Correspondencia:

*Aurora de la Peña-Díaz

E-mail: aurorad@unam.mx

0016-3813/@ 2021 Academia Nacional de Medicina de México, A.C. Publicado por Permanyer. Este es un artículo open access bajo la licencia
Fecha de recepción: 09-09-2020

Fecha de aceptación: 09-02-2021
Gac Med Mex. 2021;157(Supl3):S131-S140

Disponible en PubMed

www.gacetamedicademexico.com CC BY-NC-ND (http://creativecommons.org/licenses/by-nc-nd/4.0/). 


\section{Antecedentes}

La infección por el coronavirus 2 del síndrome respiratorio agudo grave (SARS-CoV-2) se introduce a las células a través de los receptores de la enzima convertidora de angiotensina 2 (ACE2) y se asocia con una alteración generalizada del endotelio vascular. En una interesante propuesta ${ }^{1}$, descrita por Sardu, et al., se resumen las manifestaciones clínicas que el SARS-CoV-2 puede generar en el endotelio vascular, induciendo una disfunción endotelial generalizada asociada a la pérdida, en diferente magnitud, de sus propiedades reguladoras fisiológicas, como son, entre otras, la respuesta inmunitaria, la respuesta inflamatoria, la angiogénesis, la vasodilatación y la regulación antitrombótica.

El endotelio vascular es una capa de células que constituye el revestimiento interno del sistema vascular formado por arterias, venas y capilares y se encuentra en contacto con los componentes de la sangre. Se considera un órgano endocrino que además tiene funciones que permiten controlar el tono vascular y selectivamente las diferentes moléculas de forma intravascular o extravascular y exhibe diferencias fisiológicas y morfológicas en sus diferentes segmentos y responde a muchos y diversos estímulos físicos y químicos.

Es frecuente encontrar en las diferentes publicaciones que los factores que contribuyen al deterioro del endotelio (como son diabetes, obesidad, edad e hipertensión arterial) sean factores que incrementan la gravedad de la COVID-19 y algunos de ellos resaltan como factores independientes predictivos de muerte.

Existe controversia sobre si el SARS-CoV-2 induce coagulación intravascular diseminada (CID) 0 un estado similar a $\mathrm{CID}^{2}$ (que cumple con los criterios establecidos por la Sociedad Internacional de Trombosis y Hemostasia [ISTH por sus siglas en inglés]), pero no se observa en todos los casos coagulopatía por consumo.

La sepsis induce cambios específicos diferentes en el endotelio vascular de los distintos órganos, explicando las diferencias en respuesta de estos a la estimulación inflamatoria global de la sepsis. Estos cambios inflamatorios podrían ser distintos según la causa de la sepsis y las diferencias genéticas individuales, contribuyendo a la variada expresividad clínica del síndrome ${ }^{3}$.

La infección viral puede desarrollar sepsis asociada con una disfunción orgánica. La sepsis está bien establecida como una de las causas más comunes de CID; los monocitos y las células endoteliales se activan hasta el punto de liberación de citocinas después de la lesión, con expresión del factor tisular y secreción del factor von Willebrand. La circulación de trombina libre, no controlada por anticoagulantes naturales, puede activar las plaquetas y estimular la fibrinólisis. En las últimas etapas de la COVID-19, marcadores relacionados con la fibrina (dímero $\mathrm{D}$ y productos de degradación de fibrina [PDF]) aumentan notablemente en las etapas graves y muertes, lo que sugiere una activación de la coagulación común y una condición secundaria de hiperfibrinólisis en estos pacientes ${ }^{3}$.

Las complicaciones trombóticas parecen surgir como un problema importante en pacientes con COVID-19. Se ha demostrado que alrededor del $36 \%$ de los pacientes desarrollan trombocitopenia y un $46 \%$ pueden tener dímero $D$ elevado, aunque estas tasas son aún más altas en pacientes con COVID-19 grave, entre un 57.7 y un $59.6 \%$, respectivamente ${ }^{4}$. Los niveles elevados de PDF, dímeros D, así como el tiempo de trombina prolongado se han asociado con un mal pronóstico en pacientes afectados por el nuevo coronavirus ${ }^{4}$.

El tiempo de protrombina (TP) puede encontrarse prolongado (entre el 2 y el $11 \%)^{4}$, sin embargo, en la enfermedad grave por este virus, la prolongación del TP es más evidente. El tiempo de tromboplastina parcial activado (TTPa) en los pacientes no graves se ha reportado que no hay evidencia de su alteración, pero en los pacientes graves se mostró una variación discreta ${ }^{5}$.

La prolongación de TP y la elevación del dímero $D$ y los PDF son las características principales de la coagulación anormal en esta patología, pero el TTPa no cambió significativamente. Se presume que la infección por SARS-CoV-2 puede causar daño tisular, lo que resulta en la liberación de factores tisulares, que promueven la vía de coagulación extrínseca, lo que lleva a TP prolongado. Los aumentos en el dímeros D y los PDF sugieren un estado de hiperactividad secundaria a fibrinólisis ${ }^{1}$, sin embargo, se ha documentado la presencia de fibrina reticular en el espacio extravascular y alveolar que puede ocasionar un incremento sustancial de dímeros $\mathrm{D}$ que previamente no se había visto en otras enfermedades. El valor de los dímeros $D$ en esta infección todavía es un punto de controversia, sin embargo, está claro su papel como marcador de gravedad y de mal pronóstico.

Como en otros escenarios clínicos, el laboratorio juega un papel esencial, más allá del diagnóstico etiológico, ya que permite establecer la severidad de 
la enfermedad, definir el pronóstico y seguimiento, así como ayudar en la monitorización del tratamiento. Es un punto angular en el eficiente manejo de los enfermos y esta revisión está orientada a mostrar un resumen que sirva de consulta a las diferentes disciplinas que se enfrentan y resuelven los retos que impone esta pandemia. Ofreceremos un breve resumen de las diferentes alteraciones que se identifican en el laboratorio clínico y describen en los pacientes que cursan con la fase aguda de COVID-19.

Dado que los enfermos que cursan con COVID-19 pueden manifestar trastornos en la respuesta inflamatoria, en la hemostasia, infecciones añadidas causadas por diferentes patógenos, signos de deterioro multiorgánico y/o factores de riesgo para un mal pronóstico, presentaremos las pruebas bajo esos rubros, tomando en cuenta que no es un listado exhaustivo de todas las estrategias posibles de estudio por medio de un laboratorio clínico. Las dividiremos en pruebas hematológicas (Tabla 1) y pruebas bioquímicas y especiales (Tabla 2), de manera que resulte más fácil su ubicación y comprensión. Después de un consenso entre los miembros del Comité de Trombosis y Hemostasia-AMEH A.C. que participaron en la elaboración del manuscrito, se llegó al acuerdo de presentar únicamente las pruebas que hasta la fecha han mostrado una mayor relevancia para identificar la presencia y/o severidad de la infección, las pruebas que han mostrado utilidad para identificar un pronóstico clínico o ser factores independientes de mortalidad, así como las que pueden orientar hacia la respuesta terapéutica en sus diversas opciones.

\section{Primer acuerdo}

Solicitar al laboratorio clínico:

a) Citometría hemática.

b) Química sanguínea.

c) Pruebas especiales.

\section{Segundo acuerdo}

Consideramos que durante esta contingencia es importante, salvo en protocolos de investigación, dirigir los recursos de la manera más eficiente, resaltaremos únicamente las pruebas que ofrezcan suficiente información y representen el menor costo económico.

\section{Tercer acuerdo}

Consideramos que los resultados de los diferentes estudios deben obtenerse oportunamente para poder ofrecer a los médicos clínicos la posibilidad de decisiones pertinentes durante el tratamiento de los enfermos, tratándose del curso de la COVID-19. En ocasiones la precisión de la concentración de un analito puede no ser tan relevante como poder ofrecer, oportunamente, la magnitud del incremento o decremento de un parámetro. Este comentario se dirige principalmente a los dímeros $\mathrm{D}$.

\section{Cuarto acuerdo}

Ante la ausencia de publicaciones obtenidas con población mexicana, haremos referencia a los datos obtenidos en otras poblaciones para mostrar el incremento o decremento de los parámetros sin la pretensión de que se consideren una referencia nacional o internacional.

\section{Quinto acuerdo}

Consideramos la pertinencia de introducir algunos comentarios que reflejan la experiencia de los participantes en la elaboración del manuscrito o que emanan de la asistencia a diferentes foros en los cuales expertos en el tema hicieron comunicaciones personales.

La principal manifestación que se observa en los pacientes que cursan con COVID-19 es la trombosis, que se ha reportado en todos los lechos vasculares, lo que pone de manifiesto la participación de diferentes mecanismos posibles. Sin embargo, en esta guía solo mencionaremos la pertinencia de estudiar la insuficiencia fibrinolítica, presencia de Net's, actividad protrombótica de las microvesículas y presencia de polifosfatos solo en protocolos de investigación.

El dímero $D$ se forma por acción de la plasmina sobre los depósitos de fibrina estabilizada. Su presencia se incrementa en otras condiciones clínicas, que cursan con incrementos de depósitos de fibrina de manera crónica o aguda, pero sin que necesariamente provenga de un trombo. Es decir, cualquier depósito de fibrina, aun cuando no limite el flujo en un vaso sanguíneo, incrementa el dímero $D$ como ocurre en el cáncer, los estados inflamatorios, trauma, 
Gaceta Médica de México. 2021;157(Supl 3)

Tabla 1. Pruebas hematológicas que apoyan al clínico en el seguimiento y pronóstico de los pacientes con enfermedad por coronavirus 2019

\begin{tabular}{|c|c|c|c|c|c|}
\hline Prueba & $\begin{array}{l}\text { Resultados informados } \\
\text { en estudios publicados }\end{array}$ & Objetivo & Interpretación & Referencia & Comentarios \\
\hline \multirow[t]{3}{*}{ Recuento de leucocitos } & $\begin{array}{l}\text { Severos: } 8.3 \text { (6.2-10.4 } \\
\text { x109/I) } \\
\text { Moderados: } 4.3(3.9-5.5 \\
\text { x109/I) } \\
p=0.003\end{array}$ & \multirow[t]{3}{*}{$\begin{array}{l}\text { Identificación } \\
\text { de leucocitosis } \\
\text { (coexistencia } \\
\text { con otros } \\
\text { agentes } \\
\text { patógenos) }\end{array}$} & & $\begin{array}{l}\text { Chen, et al., } \\
2020^{6}\end{array}$ & \\
\hline & $\begin{array}{l}\text { Severos: } 6.06 \mathrm{x} \\
10^{9} / \mathrm{l}(5.67-6.46) \\
\text { Moderados: } 5.07 x \\
10^{9} / 1(4.90-5.24) P<0.01\end{array}$ & & & $\begin{array}{l}\text { Bao, et al., } \\
2020^{7}\end{array}$ & \\
\hline & $\begin{array}{l}\text { Decesos: } 6.64 x \\
\text { 109/l (5.63-12.16) } \\
\text { Recuperados: } 5.605 x \\
\text { 10// (4.38-7.60) } P=0.002\end{array}$ & & & $\begin{array}{l}\text { Bonetti, } \\
\text { et al., } 2020^{8}\end{array}$ & \\
\hline \multirow[t]{3}{*}{ Recuento de neutrófilos } & $\begin{array}{l}\text { Severos: } 3.71 x \\
\text { 109// (3.36-4.06) } \\
\text { Moderados: } 5.39 x \\
\text { 10\%/l (5.22-5.55) } P<0.01\end{array}$ & \multirow{3}{*}{$\begin{array}{l}\text { Identificación } \\
\text { de neutrofilia } \\
\text { (coexistencia } \\
\text { con otros } \\
\text { agentes } \\
\text { patógenos), } \\
\text { cálculo de } \\
\text { la relación } \\
\text { neutrófilo: } \\
\text { linfocito }\end{array}$} & & $\begin{array}{l}\text { Bao, et al., } \\
2020^{7}\end{array}$ & $\begin{array}{l}\text { Punto de corte para la relación } \\
\text { neutrófilo: linfocito }=3\end{array}$ \\
\hline & $\begin{array}{l}\text { Severos: } 6.9 \text { (4.9-9.1 x } \\
\left.10^{9} / l\right) \\
\text { Moderados: } 2.7(2.1-3.7 \\
\left.x 10^{9} / l\right) \\
p=0.002\end{array}$ & & & $\begin{array}{l}\text { Chen, et al., } \\
2020^{6}\end{array}$ & \\
\hline & $\begin{array}{l}\text { Decesos: } 5.59 x \\
\text { 10// (4.45-9.72) } \\
\text { Recuperados: } 4.13 x \\
10^{9} / 1(2.71-5.79) P<0.001\end{array}$ & & & $\begin{array}{l}\text { Bonetti, } \\
\text { et al., } 2020^{8}\end{array}$ & \\
\hline \multirow[t]{4}{*}{ Recuento de linfocitos } & $\begin{array}{l}\text { Valores }<0.8 \times 10^{9} / l \\
72 \% \text { de casos severos } \\
10 \% \text { de casos moderados } \\
p=0.008\end{array}$ & \multirow[t]{4}{*}{$\begin{array}{l}\text { Identificación de } \\
\text { la linfopenia } \\
\text { Cálculo de } \\
\text { la relación } \\
\text { neutrófilo: } \\
\text { linfocito }\end{array}$} & & $\begin{array}{l}\text { Chen, et al., } \\
2020^{6}\end{array}$ & $\begin{array}{l}\text { En pacientes de } 6 \text { días a } \\
21 \text { años, la linfopenia fue el } \\
\text { hallazgo de laboratorio más } \\
\text { consistente, pero sin valor } \\
\text { pronóstico. Se observaron } \\
\text { marcadores inflamatorios } \\
\text { significativamente elevados } \\
\text { que sugieren un estado } \\
\text { hiperinflamatorio en pacientes } \\
\text { con enfermedad grave, como } \\
\text { se describe en adultos }\end{array}$ \\
\hline & & & & $\begin{array}{l}\text { Zachariah, } \\
\text { et al., } 2020^{9}\end{array}$ & \\
\hline & $\begin{array}{l}\text { Severos: } 0.80 x \\
10^{9} / l(0.75-0.84) \\
\text { Moderados: } 1.15 x \\
10^{9} / 1(1.08-1.22) P<0.01\end{array}$ & & & $\begin{array}{l}\text { Bao, et al., } \\
2020^{7}\end{array}$ & \\
\hline & $\begin{array}{l}\text { Decesos: } 0.75 x \\
10^{9} / /(0.56-1.01) \\
\text { Recuperados: } 1.04 x \\
10^{9} / /(0.81-1.30) P<0.001\end{array}$ & & & $\begin{array}{l}\text { Bonetti, } \\
\text { et al., } 2020^{8}\end{array}$ & \\
\hline $\begin{array}{l}\text { Tiempo de } \\
\text { protrombina (TP) }\end{array}$ & $\begin{array}{l}\text { Severos: } 14.6 \text { s (13.6-14.6) } \\
\text { Moderados: } \\
\text { 13.4 (12.8-13.7) } P=0.15\end{array}$ & $\begin{array}{l}\text { Identificación del } \\
\text { surgimiento de } \\
\text { coagulopatía }\end{array}$ & $\begin{array}{l}\text { Presencia } \\
\text { de una } \\
\text { coagulopatía } \\
\text { por consumo o } \\
\text { lesión hepática } \\
\text { Valor de } \\
\text { referencia: } \\
\text { 11.5-14.5 s }\end{array}$ & $\begin{array}{l}\text { Chen, et al., } \\
2020^{6}\end{array}$ & $\begin{array}{l}\text { Debe informarse } \\
\text { exclusivamente en segundos, } \\
\text { cambios sutiles no se aprecian } \\
\text { en el informe del INR o como } \\
\text { cociente con el control. }\end{array}$ \\
\hline
\end{tabular}


Tabla 1. Pruebas hematológicas que apoyan al clínico en el seguimiento y pronóstico de los pacientes con enfermedad por coronavirus 2019 (Continuation)

\begin{tabular}{|c|c|c|c|c|c|}
\hline Prueba & $\begin{array}{l}\text { Resultados informados } \\
\text { en estudios publicados }\end{array}$ & Objetivo & Interpretación & Referencia & Comentarios \\
\hline & $\begin{array}{l}\text { Severos: } \\
17.32 \mathrm{~s}(13.7-20.7)\end{array}$ & & $\begin{array}{l}\text { Valor de } \\
\text { referencia: } \\
11.9-14.4 \mathrm{~s}\end{array}$ & $\begin{array}{l}\text { Zachariah, } \\
\text { et al., } 2020^{9}\end{array}$ & $\begin{array}{l}\text { Pacientes de } 6 \text { días a } 21 \text { años. } \\
\text { No se obtuvieron valores } \\
\text { consistentes en pacientes } \\
\text { con enfermedad moderada. } \\
\text { El valor del TP puede variar } \\
\text { dependiendo del reactivo }\end{array}$ \\
\hline & $\begin{array}{l}\text { Severos: } 12.80 \\
\text { s (12.29-13.30) } \\
\text { Moderados: } \\
12.45 \text { s (11.98-12.91) }\end{array}$ & & & $\begin{array}{l}\text { Bao, et al., } \\
2020^{7}\end{array}$ & \\
\hline & $\begin{array}{l}\text { Decesos: INR } \\
1.12(1.03-1.28) \\
\text { Recuperados: INR } \\
1.04(1.00-1.10) P=0.001\end{array}$ & & & & \\
\hline \multirow[t]{4}{*}{$\begin{array}{l}\text { Tiempo de } \\
\text { tromboplastina parcial } \\
\text { activado (TTPa) }\end{array}$} & $\begin{array}{l}\text { Severos: } 33.7 \mathrm{~s}(32.1-38.4) \\
\text { Moderados: } \\
44.0 \mathrm{~s}(42.6-47.6) \\
P=0.002\end{array}$ & $\begin{array}{l}\text { Identificación del } \\
\text { surgimiento de } \\
\text { coagulopatía }\end{array}$ & $\begin{array}{l}\text { Reflejar la } \\
\text { actividad de } \\
\text { la heparina } \\
\text { convencional } \\
\text { Presencia } \\
\text { de una } \\
\text { coagulopatía } \\
\text { por consumo } \\
\text { Se ha } \\
\text { informado } \\
\text { que puede } \\
\text { presentarse un } \\
\text { incremento que } \\
\text { puede sugerir } \\
\text { la presencia de } \\
\text { anticoagulante } \\
\text { lúpico } \\
\text { Valor de } \\
\text { referencia: } \\
29.0-42.0 \text { s }\end{array}$ & $\begin{array}{l}\text { Chen, et al., } \\
2020^{6}\end{array}$ & $\begin{array}{l}\text { Los resultados prolongados } \\
\text { del TTPa en estas condiciones } \\
\text { pueden ser reflejo de un estado } \\
\text { inflamatorio generalizado, } \\
\text { presencia de heparina. } \\
\text { Recomendamos hacer pruebas } \\
\text { para confirmar la presencia de } \\
\text { un inhibidor lúpico }\end{array}$ \\
\hline & $\begin{array}{l}\text { Severos: } \\
47.77 \text { s (32.4-108.5) }\end{array}$ & & $\begin{array}{l}\text { Valor de } \\
\text { referencia: } \\
23.9-34.7 \text { s }\end{array}$ & $\begin{array}{l}\text { Zachariah, } \\
\text { et al., } 2020^{9}\end{array}$ & $\begin{array}{l}\text { Pacientes de } 6 \text { días a } 21 \text { años. } \\
\text { No se obtuvieron valores } \\
\text { consistentes en pacientes } \\
\text { con enfermedad moderada. } \\
\text { El valor del TTPa puede variar } \\
\text { dependiendo del reactivo }\end{array}$ \\
\hline & $\begin{array}{l}\text { Severos: } \\
32.92(30.78-35.06) \\
\text { Moderados: } \\
33.49(31.17-35.82)\end{array}$ & & & $\begin{array}{l}\text { Bao, et al., } \\
2020^{7}\end{array}$ & \\
\hline & $\begin{array}{l}\text { Decesos: } 34 \mathrm{~s} \text { (29-37) } \\
\text { Recuperados: } 31 \mathrm{~s}(29-34) \\
P=0.014\end{array}$ & & & $\begin{array}{l}\text { Bonetti, } \\
\text { et al., } 2020^{8}\end{array}$ & \\
\hline Fibrinógeno & & $\begin{array}{l}\text { Identificación de } \\
\text { la coagulopatía } \\
\text { (sobre todo si es } \\
\text { de consumo) }\end{array}$ & $\begin{array}{l}\text { Identificar } \\
\text { coagulopatía } \\
\text { por consumo }\end{array}$ & & $\begin{array}{l}\text { El incremento se asocia } \\
\text { a proceso inflamatorio y } \\
\text { respuesta de fase aguda. } \\
\text { Cualquier decremento sugiere } \\
\text { una coagulopatía por } \\
\text { consumo, CID }\end{array}$ \\
\hline
\end{tabular}


Tabla 1. Pruebas hematológicas que apoyan al clínico en el seguimiento y pronóstico de los pacientes con enfermedad por coronavirus 2019 (Continuation)

\begin{tabular}{|c|c|c|c|c|c|}
\hline Prueba & $\begin{array}{l}\text { Resultados informados } \\
\text { en estudios publicados }\end{array}$ & Objetivo & Interpretación & Referencia & Comentarios \\
\hline \multirow[t]{4}{*}{ Dímero D } & $\begin{array}{l}\text { Severos: } \\
2.6 \mu \mathrm{g} / \mathrm{ml}(0.6-18.7) \\
\text { Moderados: } \\
0.3 \mu \mathrm{g} / \mathrm{ml}(0.3-0.4) \\
p=0.029\end{array}$ & \multirow[t]{4}{*}{$\begin{array}{l}\text { Identificación de } \\
\text { coagulopatía de } \\
\text { consumo }\end{array}$} & $\begin{array}{l}\text { Identificar una } \\
\text { coagulopatía } \\
\text { de consumo } \\
\text { Identificar } \\
\text { incompetencia } \\
\text { fibrinolítica } \\
\text { Identificar la } \\
\text { edad de los } \\
\text { trombos de } \\
\text { los cuales } \\
\text { provienen los } \\
\text { fragmentos } \\
\text { Marcador } \\
\text { independiente } \\
\text { de mortalidad } \\
\text { Valor de } \\
\text { referencia: } \\
<0.5 \mu \text { g/ml }\end{array}$ & $\begin{array}{l}\text { Chen, et al., } \\
2020^{6}\end{array}$ & $\begin{array}{l}\text { Se debe solicitar al séptimo día } \\
\text { de inicio de los síntomas y dar } \\
\text { un seguimiento, entre } 24 \text { y } 48 \text { h, } \\
\text { sin que se solicite en menos de } \\
24 \text { h, para orientar el desenlace } \\
\text { clínico del paciente } \\
\text { Metodología cuantitativa sin } \\
\text { detrimento del tiempo en que } \\
\text { se obtenga el resultado } \\
\text { La magnitud entre } 3 \text { a } 6 \text { veces } \\
\text { del incremento con respecto } \\
\text { al resultado basal es un factor } \\
\text { de riesgo independiente para } \\
\text { mortalidad }\end{array}$ \\
\hline & $\begin{array}{l}\text { Severos: } \\
4.87 \mu \mathrm{g} / \mathrm{ml}(0.95-18.775)\end{array}$ & & \multirow[t]{3}{*}{$\begin{array}{l}\text { Valor de } \\
\text { referencia: } \\
\leq 0.5 \mu \mathrm{g} / \mathrm{ml}\end{array}$} & $\begin{array}{l}\text { Zachariah, } \\
\text { et al., } 2020^{9}\end{array}$ & \multirow[t]{3}{*}{$\begin{array}{l}\text { Pacientes de } 6 \text { días a } 21 \text { años. } \\
\text { No se obtuvieron valores } \\
\text { consistentes en pacientes con } \\
\text { enfermedad moderada }\end{array}$} \\
\hline & $\begin{array}{l}\text { Severos: } 1.29(0.03-0.54) \\
\text { Moderados: } \\
0.47(0.40-0.53) \\
p \leq 0.01\end{array}$ & & & $\begin{array}{l}\text { Bao, et al., } \\
2020^{7}\end{array}$ & \\
\hline & $\begin{array}{l}\text { Decesos: } \\
1.99 \mathrm{~g} / \mathrm{l}(1.27-3.63) \\
\text { Recuperados: } \\
0.91 \mathrm{~g} / \mathrm{l}(0.60-2.03) \\
P<0.001\end{array}$ & & & $\begin{array}{l}\text { Bonetti, } \\
\text { et al., } 2020^{8}\end{array}$ & \\
\hline
\end{tabular}

INR: índice internacional normalizado.

ruptura de un órgano, el embarazo, la sepsis, el daño hepático, el daño cardiaco, la cirugía reciente, la hemodiálisis y la CID.

Los valores de linfopenia y la neutrofilia son consecuencia de la expresión de la ACE2, principal receptor del SARS-CoV-2 en la superficie celular de los linfocitos. Su disminución gradual en la circulación puede estar asociada con la disfunción linfocítica e inmunosupresión, lo que contribuye a que los pacientes tengan un mayor riesgo de coinfecciones y un peor pronóstico. También es importante observar la asociación de valores elevados de proteína $C$ reactiva y neutrofilia en los decesos por COVID-19, ya que ambos parámetros reflejan la presencia de una viremia con una posible coinfección bacteriana, que frecuentemente complica el curso clínico de la enfermedad con un pronóstico desfavorable ${ }^{10}$

El incremento de los valores de lactato deshidrogenasa (LDH) refleja el alcance de la lesión pulmonar y de otros órganos en pacientes con COVID-19 ${ }^{10}$.

\section{Conclusiones}

Finalmente, el Comité de Trombosis y Hemostasia, con base en la evidencia, realiza una serie de recomendaciones que considerar en los pacientes con COVID-19 a efecto de ayudar al clínico en la mejor toma de decisión para evolución y tratamiento.

La infección por el SARS-CoV-2, que produce COVID-19, ha generado más preguntas que 
Tabla 2. Pruebas bioquímicas y otras pruebas que apoyan al clínico en el seguimiento y pronóstico de los pacientes con enfermedad por coronavirus 2019

\begin{tabular}{|c|c|c|c|c|c|}
\hline Prueba & $\begin{array}{l}\text { Resultados informados en } \\
\text { estudios publicados }\end{array}$ & Objetivo & Interpretación & Referencia & Comentarios \\
\hline \multirow[t]{4}{*}{ Proteína $\mathrm{C}$ reactiva } & $\begin{array}{l}\text { Severos: } 139.4 \mathrm{mg} / \mathrm{l}(86.9-165.1) \\
\text { Moderados: } 22 \mathrm{mg} / \mathrm{l}(14.7-119.4) \\
p=0.003\end{array}$ & \multirow[t]{4}{*}{$\begin{array}{l}\text { Monitorización } \\
\text { de infección } \\
\text { y respuesta } \\
\text { inflamatoria }\end{array}$} & \multirow[t]{2}{*}{$\begin{array}{l}\text { Valores elevados en } \\
\text { procesos inflamatorios, } \\
\text { algunos trastornos } \\
\text { autoinmunes y } \\
\text { enfermedades de las } \\
\text { arterias coronarias. } \\
\text { Valor de referencia } \\
<1 \mathrm{mg} / \mathrm{l}\end{array}$} & $\begin{array}{l}\text { Chen, et al., } \\
2020^{6}\end{array}$ & $\begin{array}{l}\text { El } 100 \% \text { de los } \\
\text { pacientes severos } \\
\text { tenían valores } \\
\text { superiores a } \\
60 \text { mg/l, únicamente } \\
\text { el } 33.3 \% \text { de los } \\
\text { moderados, } \\
P=0.002\end{array}$ \\
\hline & $\begin{array}{l}\text { Severos: } 60.91 \mathrm{mg} / \mathrm{l}(49.24-72.5) \\
\text { Moderados: } 19.83 \mathrm{mg} / \mathrm{l}(16.67-23.0) \\
P<0.01\end{array}$ & & & $\begin{array}{l}\text { Bao, et al., } \\
2020^{7}\end{array}$ & \\
\hline & $\begin{array}{l}\text { Severos: } 18.825 \mathrm{mg} / \mathrm{dl}(12.69-25.78) \\
\text { Moderados: } 4.45 \mathrm{mg} / \mathrm{dl}(0.027-25.7) \\
P<0.001\end{array}$ & & \multirow[t]{2}{*}{$\begin{array}{l}\text { Valor de referencia } \\
\leq 10 \mathrm{mg} / \mathrm{dl}\end{array}$} & $\begin{array}{l}\text { Zachariah, } \\
\text { et al., } 2020^{9}\end{array}$ & \\
\hline & $\begin{array}{l}\text { Decesos: } 165.65 \mathrm{~g} / \mathrm{l}(82.45-241.4) \\
\text { Recuperados: } 60.3 \mathrm{~g} / \mathrm{l}(23.6-154.4) \\
P<0.001\end{array}$ & & & $\begin{array}{l}\text { Bonetti, } \\
\text { et al., } 2020^{8}\end{array}$ & $\begin{array}{l}\text { Pacientes de } 6 \text { días } \\
\text { a } 21 \text { años }\end{array}$ \\
\hline \multirow[t]{3}{*}{ Ferritina } & $\begin{array}{l}\text { Severos: } \\
\text { 1,598.2 } \mu \mathrm{g} / \mathrm{l}(1,426.6-2,030.0) \\
\text { Moderados: } \\
337.4 \mu \mathrm{g} / \mathrm{l}(282.2-1,275.4) P=0.049\end{array}$ & \multirow[t]{3}{*}{$\begin{array}{l}\text { Monitorización } \\
\text { de la infección/ } \\
\text { respuesta } \\
\text { inflamatoria }\end{array}$} & $\begin{array}{l}\text { Niveles elevados en } \\
\text { inflamación aguda o } \\
\text { crónica, el consumo } \\
\text { crónico de alcohol, } \\
\text { enfermedad hepática, } \\
\text { insuficiencia renal, } \\
\text { síndrome metabólico, } \\
\text { o malignidad en lugar } \\
\text { de ser causados por la } \\
\text { sobrecarga de hierro. } \\
\text { Valor de referencia } \\
30-400 \mu \text { g/l }\end{array}$ & $\begin{array}{l}\text { Chen, et al., } \\
2020^{6}\end{array}$ & $\begin{array}{l}\text { El } 100 \% \text { de los } \\
\text { pacientes severos } \\
\text { tenían valores } \\
\text { mayores a } 800 \mu \mathrm{g} / \mathrm{l} \text {, } \\
\text { mientras que en } \\
\text { solo el } 30 \% \text { de } \\
\text { los moderados su } \\
\text { valor era superior a } \\
800 \mu \mathrm{g} / \mathrm{l}, P=0.003\end{array}$ \\
\hline & Severos: 435 ng/ml $(178-1,374)$ & & \multirow[t]{2}{*}{$\begin{array}{l}\text { Valor de referencia } \\
\leq 150 \mathrm{ng} / \mathrm{ml}\end{array}$} & $\begin{array}{l}\text { Zachariah, } \\
\text { et al., } 2020^{9}\end{array}$ & $\begin{array}{l}\text { Pacientes de } 6 \\
\text { días a } 21 \text { años. } \\
\text { No se obtuvieron } \\
\text { valores consistentes } \\
\text { en pacientes } \\
\text { con enfermedad } \\
\text { moderada }\end{array}$ \\
\hline & $\begin{array}{l}\text { Decesos: } 1,285 \mathrm{ng} / \mathrm{ml}(431-2,409) \\
\text { Recuperados: } \\
701.5 \mathrm{ng} / \mathrm{ml}(382-1,475) P=0.01\end{array}$ & & & $\begin{array}{l}\text { Bonetti, } \\
\text { et al., } 2020^{8}\end{array}$ & \\
\hline \multirow[t]{3}{*}{ Procalcitonina } & $\begin{array}{l}\text { Severos: } 0.18 \mathrm{ng} / \mathrm{ml}(0.13-0.81) \\
\text { Moderados: } 0.05 \mathrm{ng} / \mathrm{ml}(0.04-0.06) \\
p=0.059\end{array}$ & \multirow[t]{3}{*}{$\begin{array}{l}\text { Identificación } \\
\text { de } \\
\text { coinfecciones } \\
\text { bacterianas }\end{array}$} & \multirow[t]{2}{*}{$\begin{array}{l}\text { Identificar coexistencia } \\
\text { de infecciones } \\
\text { bacterianas } \\
\text { Valor de referencia } \\
0.02-0.05 \mathrm{ng} / \mathrm{ml}\end{array}$} & $\begin{array}{l}\text { Chen, et al., } \\
2020^{6}\end{array}$ & $\begin{array}{l}\text { A menos que } \\
\text { sea clínicamente } \\
\text { justificado, la } \\
\text { evaluación no } \\
\text { debería ser } \\
\text { reordenada en } \\
\text { menos de } \\
24 \text { horas }\end{array}$ \\
\hline & $\begin{array}{l}\text { Severos: } 0.14 \mathrm{ng} / \mathrm{ml}(0.11-0.17) \\
\text { Moderados: } 0.07 \mathrm{ng} / \mathrm{ml}(0.05-0.09) \\
p<0.01\end{array}$ & & & $\begin{array}{l}\text { Bao, et al., } \\
2020^{7}\end{array}$ & \\
\hline & $\begin{array}{l}\text { Severos: } 5.3 \mathrm{ng} / \mathrm{ml}(0.13-29.89) \\
\text { Moderados: } 0.74 \text { ng/ml (0.04-7.44) }\end{array}$ & & $\begin{array}{l}\text { Valor de } \\
\text { referencia } \leq 0.08 \mathrm{ng} / \mathrm{ml}\end{array}$ & $\begin{array}{l}\text { Zachariah, } \\
\text { et al., } 2020^{9}\end{array}$ & $\begin{array}{l}\text { Pacientes de } 6 \text { días } \\
\text { a } 21 \text { años }\end{array}$ \\
\hline
\end{tabular}


Gaceta Médica de México. 2021;157(Supl 3)

Tabla 2. Pruebas bioquímicas y otras pruebas que apoyan al clínico en el seguimiento y pronóstico de los pacientes con enfermedad por coronavirus 2019 (Continuation)

\begin{tabular}{|c|c|c|c|c|c|}
\hline Prueba & $\begin{array}{l}\text { Resultados informados en } \\
\text { estudios publicados }\end{array}$ & Objetivo & Interpretación & Referencia & Comentarios \\
\hline \multirow[t]{3}{*}{ LDH } & $\begin{array}{l}\text { Severos: } 537.0 \mathrm{U} / \mathrm{I}(433.5-707.5) \\
\text { Moderados: } 224.0 \mathrm{U} / \mathrm{I}(200.3-251.8) \\
p=0.001\end{array}$ & \multirow[t]{3}{*}{$\begin{array}{l}\text { Identificación } \\
\text { de daño } \\
\text { pulmonar } \\
\text { y/o falla } \\
\text { multiorgánica }\end{array}$} & \multirow[t]{3}{*}{$\begin{array}{l}\text { Daño pulmonar y falla } \\
\text { multiorgánica Valor de } \\
\text { Referencia 135-225 U/l }\end{array}$} & $\begin{array}{l}\text { Chen, et al., } \\
2020^{6}\end{array}$ & $\begin{array}{l}\text { El } 90.9 \% \text { de los } \\
\text { pacientes severos } \\
\text { presentaron } \\
\text { valores }>300 \mathrm{U} / \mathrm{l} \\
P=0.000\end{array}$ \\
\hline & $\begin{array}{l}\text { Severos: } 344.48 \text { U/I (307.08-381.88) } \\
\text { Moderados: } 224.20 \\
\text { U/I (205.33-243.07) }\end{array}$ & & & $\begin{array}{l}\text { Bao, et al., } \\
2020^{7}\end{array}$ & \\
\hline & $\begin{array}{l}\text { Decesos: } 521 \mathrm{U} / \mathrm{l}(416-636) \\
\text { Recuperados: } 316 \\
\text { U/I (247.0-397.25) } P<0.001\end{array}$ & & & $\begin{array}{l}\text { Bonetti, } \\
\text { et al., } 2020^{8}\end{array}$ & \\
\hline \multirow[t]{3}{*}{ Transaminasas } & $\begin{array}{l}\text { Severos: AST } 47.0 \mathrm{U} / \mathrm{I}(28.0-74.5) \\
\text { ALT 42.0 U/I (32.5-50) } \\
\text { Moderados: AST 24.0 } \\
\text { U/I (21.5-26.5) ALT } 16.0 \\
\text { U/I (13.3-21.8) } \\
\text { AST } P=0.014 \\
\text { ALT } P=0.000\end{array}$ & \multirow[t]{3}{*}{$\begin{array}{l}\text { Identificación } \\
\text { de daño } \\
\text { hepático }\end{array}$} & \multirow[t]{3}{*}{$\begin{array}{l}\text { Daño hepático } \\
\text { Valores de referencia } \\
\text { ALT } 41 \mathrm{U} / \mathrm{U} \\
\text { AST } 40 \mathrm{U} / \mathrm{l}\end{array}$} & $\begin{array}{l}\text { Chen, et al., } \\
2020^{6}\end{array}$ & \\
\hline & $\begin{array}{l}\text { Severos: AST: } 36.78 \\
\text { U/I (33.69-39.87) } \\
\text { ALT: 33.78 U/I (29.54-38.0) } \\
\text { Moderados: AST: 26.24 } \\
\text { U/I (25.29-28.18) } \\
\text { ALT: } 24.85 \text { U/I (22.69-27.0) } \\
\text { ALT<0.01 }\end{array}$ & & & $\begin{array}{l}\text { Bao, et al., } \\
2020^{7}\end{array}$ & \\
\hline & $\begin{array}{l}\text { Decesos: AST } 61.5 \text { U/I (44-83) } \\
\text { ALT } 34 \text { U/I (23-49) } \\
\text { Recuperados: AST } 45 \text { U/I (25-49) } \\
\text { ALT } 31.5 \text { U/l (21-46) } \\
\text { AST } P=0.001 \\
\text { ALT } P=0.516\end{array}$ & & & $\begin{array}{l}\text { Bonetti, } \\
\text { et al., } 2020^{8}\end{array}$ & \\
\hline \multirow[t]{3}{*}{ Bilirrubina } & $\begin{array}{l}\text { Severos: } 8.8 \mathrm{mmol} / \mathrm{l}(7.9-10.5) \\
\text { Moderados: } 7.8 \mathrm{mmol} / \mathrm{l}(6.4-9-5)\end{array}$ & \multirow[t]{3}{*}{$\begin{array}{l}\text { Identificación } \\
\text { de daño } \\
\text { hepático }\end{array}$} & \multirow[t]{3}{*}{$\begin{array}{l}\text { Daño hepático } \\
\text { Valor de referencia } \leq 26 \\
\mathrm{mmol} / \mathrm{l}\end{array}$} & $\begin{array}{l}\text { Chen, et al., } \\
2020^{6}\end{array}$ & \\
\hline & $\begin{array}{l}\text { Severos: } 11.86 \mathrm{mmol} / /(10.81-12.91 \\
\text { Moderados: } 10.38 \\
\mathrm{mmol} / \mathrm{l}(9.78-10.99)\end{array}$ & & & $\begin{array}{l}\text { Bao, et al., } \\
2020^{7}\end{array}$ & \\
\hline & $\begin{array}{l}\text { Decesos: } 11.7 \mu \mathrm{mol} / /(8.7-19.5) \\
\text { Recuperados: } 10.6 \mu \mathrm{mol} / /(8.7-13.9) \\
P=0.109\end{array}$ & & & $\begin{array}{l}\text { Bonetti, } \\
\text { et al., } 2020^{8}\end{array}$ & \\
\hline \multirow[t]{3}{*}{ Albúmina } & $\begin{array}{l}\text { Severos: } 29.6 \mathrm{~g} / \mathrm{l}(28.6-33.0) \\
\text { Moderados: } 37.2 \mathrm{~g} / \mathrm{l}(35.8-38.8) \\
P=0.013\end{array}$ & \multirow[t]{3}{*}{$\begin{array}{l}\text { Identificación } \\
\text { de insuficiencia } \\
\text { hepática }\end{array}$} & \multirow[t]{3}{*}{$\begin{array}{l}\text { Daño hepático } \\
\text { Valor de referencia } \\
35.0-52.0 \mathrm{~g} / \mathrm{l}\end{array}$} & $\begin{array}{l}\text { Chen, et al., } \\
2020^{6}\end{array}$ & $\begin{array}{l}\text { El } 63.6 \% \text { de } \\
\text { los pacientes } \\
\text { moderados } \\
\text { presentaron } \\
\text { valores }<32.0 \mathrm{~g} / \mathrm{l} \\
P=0.024\end{array}$ \\
\hline & $\begin{array}{l}\text { Severos: } 34.29 \mathrm{~g} / \mathrm{l}(32.79-35.80) \\
\text { Moderados: } 39.41 \mathrm{~g} / \mathrm{l}(37.95-40.87 \\
P<0.01\end{array}$ & & & $\begin{array}{l}\text { Bao, et al., } \\
2020^{7}\end{array}$ & \\
\hline & $\begin{array}{l}\text { Decesos: } 34.6 \mathrm{~g} / \mathrm{l}(31.8-35.9) \\
\text { Recuperados: } 36.5 \mathrm{~g} / \mathrm{l}(33.8-39.3) \\
P<0.001\end{array}$ & & & $\begin{array}{l}\text { Bonetti, } \\
\text { et al., } 2020^{8}\end{array}$ & \\
\hline
\end{tabular}


Tabla 2. Pruebas bioquímicas y otras pruebas que apoyan al clínico en el seguimiento y pronóstico de los pacientes con enfermedad por coronavirus 2019 (Continuation)

\begin{tabular}{|c|c|c|c|c|c|}
\hline Prueba & $\begin{array}{l}\text { Resultados informados en } \\
\text { estudios publicados }\end{array}$ & Objetivo & Interpretación & Referencia & Comentarios \\
\hline \multirow[t]{2}{*}{ Urea } & $\begin{array}{l}\text { Severos: } 5.59 \mathrm{mmol} / \mathrm{l}(5.39-6.51) \\
\text { Moderados: } 4.36 \mathrm{mmol} / \mathrm{l}(4.12-4.59) \\
P<0.01\end{array}$ & \multirow[t]{2}{*}{$\begin{array}{l}\text { Identificación } \\
\text { daño renal }\end{array}$} & & $\begin{array}{l}\text { Bao, et al., } \\
2020^{7}\end{array}$ & \\
\hline & $\begin{array}{l}\text { Decesos: } 23.4 \mathrm{mmol} / / \text { (16.1-33.6) } \\
\text { Recuperados: } 12.1 \\
\mathrm{mmol} / \mathrm{l}(10.4-19.3) P<0.001\end{array}$ & & Daño renal & $\begin{array}{l}\text { Bonetti, } \\
\text { et al., } 2020^{8}\end{array}$ & \\
\hline \multirow[t]{3}{*}{ Creatinina } & $\begin{array}{l}\text { Severos: } 82.0 \mu \mathrm{mol} / /(67.5-91.5) \\
\text { Moderados: } 76.5 \mu \mathrm{mol} / \mathrm{l}(63.3-81.0)\end{array}$ & \multirow[t]{3}{*}{$\begin{array}{l}\text { Identificación } \\
\text { daño renal }\end{array}$} & \multirow[t]{3}{*}{$\begin{array}{l}\text { Daño renal } \\
\text { Valor de referencia } \\
59-104 \mu \mathrm{mol} / \mathrm{l}\end{array}$} & $\begin{array}{l}\text { Chen, et al., } \\
2020^{6}\end{array}$ & \\
\hline & $\begin{array}{l}\text { Severos: } 72.94 \mu \mathrm{mol} / \mathrm{l}(69.23-76.66) \\
\text { Moderados: } 66.97 \\
\mu \mathrm{mol} / /(64.65-69.28)\end{array}$ & & & $\begin{array}{l}\text { Bao, et al., } \\
2020^{7}\end{array}$ & \\
\hline & $\begin{array}{l}\text { Decesos: } 124 \mu \mathrm{mol} / / \mathrm{l}(85-155) \\
\text { Recuperados: } 91 \mu \mathrm{mol} / /(73-104) \\
P<0.001\end{array}$ & & & $\begin{array}{l}\text { Bonetti, } \\
\text { et al., } 2020^{8}\end{array}$ & \\
\hline Troponina & $\begin{array}{l}\text { Decesos: } 47.5 \mathrm{ng} / \mathrm{l}(2.0-90.0) \\
\text { Recuperados: } 8.0 \mathrm{ng} / \mathrm{l}(5.1-15.0) \\
P<0.001\end{array}$ & $\begin{array}{l}\text { Identificación } \\
\text { daño cardiaco }\end{array}$ & Daño cardiaco & $\begin{array}{l}\text { Bonetti, } \\
\text { et al., } 2020^{8}\end{array}$ & \\
\hline Glucosa & $\begin{array}{l}\text { Decesos: } 7.63 \mathrm{mmol} / \mathrm{l}(6.44-10.60) \\
\text { Recuperados: } 6.10 \\
\mathrm{mmol} / \mathrm{l}(5.61-6.77) P<0.001\end{array}$ & $\begin{array}{l}\text { Identificación } \\
\text { desarreglo } \\
\text { alteración } \\
\text { metabólica }\end{array}$ & $\begin{array}{l}\text { Identificar presencia de } \\
\text { diabetes }\end{array}$ & $\begin{array}{l}\text { Bonetti, } \\
\text { et al., } 2020^{8}\end{array}$ & \\
\hline
\end{tabular}

respuestas debido a su afectación multisistémica, endotelial e inmunitaria. El clínico que se enfrenta al paciente con COVID-19 en sus diferentes etapas de infección tiene el enorme reto de correlacionar la historia clínica con los diferentes parámetros de laboratorio que han sido informados en los diversos estudios clínicos. Específicamente, en los parámetros de laboratorio han surgido algunos que adquieren particular relevancia, como marcadores de progresión de la enfermedad, tales como linfopenia, relación neutrófilo:linfocito, concentración de ferritina, proteína $C$ reactiva y dímeros $\mathrm{D}$. Este último parámetro sigue siendo motivo de investigación debido a las implicaciones clínicas y pronósticas y en la toma de decisión del clínico con respecto a la dosis de la terapia anticoagulante, sin embargo, su incremento es un indicador de progresión de la enfermedad.

\section{Conflicto de intereses}

Ninguno de los autores tiene conflicto de intereses. El Comité de Trombosis y Hemostasia- AMEH. A.C. es una Sociedad Civil sin fines de lucro.

\section{Financiamiento}

Ninguno de los autores recibió financiamiento.

\section{Responsabilidades éticas en el manuscrito}

Los autores declaran que en esta investigación no se han realizado experimentos en seres humanos ni en animales. Tampoco se han manejado datos de pacientes propios de las instituciones a los cuales están afiliados. Se trata de un artículo de revisión donde se señala el aporte suministrado por las evidencias publicadas, adecuadamente referenciadas.

\section{Bibliografía}

1. Sardu C, Gambardella J, Morelli M, Wang X, Marfella R, Santulli G. Hipertension, thrombosis, kidney failure, and diabetes: Is COVID19 an endothelial disease? A comprenhensive evaluation of clinical and basic evidence. J Clin Med. 2020;9(5):1417.

2. Panigada M, Bottino N, Tagliabue $P$, Novembrino C, Chantarangkul V, Pesenti $F$, et al. Hypercoagulability of COVID-19 patients in intensive care unit: A report of thromboelastography findings and other parameters of hemostasis. J Thromb Haemost. 2020;18(7):1738-42.

3. Tang N, Li D, Wang X, Sun Z. Abnormal coagulation parameters are associated with poor prognosis in patients with novel coronavirus pneumonia. J Thromb Haemost. 2020;18:844-7. 
Gaceta Médica de México. 2021;157(Supl 3)

4. Giannis D, Ziogas L, Gianni P. Coagulation disorders in coronavirus infected patients: COVID 19, SARS-T-Cov-1, MERS-CoV and lessons from the past. J Clin Vir. 2020;127:104362.

5. Violi F, Pastori D, Cangemi R, Loffredo L. Hypercoagulation and antithrombotic treatment in coronavirus 2019: A new challenge. Thromb Haemost. 2020;120:949-56.

6. Chen G, Wu D, Guo W, Cao Y, Huang D, Wang H, et al. Clinical and immunological features of severe and moderate coronavirus disease 2019. J Clin Invest. 2020;130(5):2620-9.

7. Bao J, Li C, Zhang K, Kang H, Chen W, Gu B. Comparative analysis of laboratory indexes of severe and non severe patients infected with $\mathrm{CO}$ VID-19. Clin Chem Acta. 2020;509:180-94.
8. Bonetti G, Manelli F, Patroni A. Laboratory predictors of death from coronavirus disease 2019 (COVID-19) in the area of Valcamonica, Italy. Clin Chem Lab Med. 2020;58(7):1100-05.

9. Zachariah P, Johnson C, Halabi K. Epidemiology, clinical features and disease severity in patients with coronavirus disease 2019 (COVID-19) in a Children's Hospital in New York City. JAMA Pediatr. 2020;174:e202430.

10. Li Y, Hu Y, Yu J, Ma T. Retrospective analysis of laboratory testing in 54 patients with severe or critical type 2019 novel coronavirus pneumonia. Lab Invest. 2020;100:794-800. 